$15^{\text {th }}$ International Conference on

AEROSPACE SCIENCES \& AVIATION TECHNOLOGY,

ASAT - 15 - May 28 - 30, 2013, Email: asat@mtc.edu.eg,

Military Technical College, Kobry Elkobbah, Cairo, Egypt,

Tel: +(202) $24025292-24036138$, Fax: + +(202) 22621908

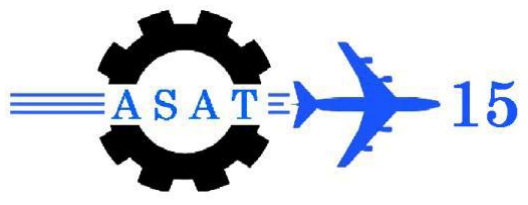

\title{
Trajectory Modeling of GRAD Rocket with Low-Cost Terminal Guidance Upgrade Coupled to Range Increase Through Step-Like Thrust-Curves
}

\author{
Florin Mingireanu
}

\begin{abstract}
The paper shows a full 6 DOF modeling for a GRAD rocket in Earth's non-inertial frame. For numerical purposes the modeling is limited to flat Earth approximation. The full motion equations are shown and all terms are explained together with the aerodynamics parameters for the entire flight envelope. Non-linear interpolation is used for aerodynamic coefficients and their derivatives. We present the typical dispersion factors due to rocket production inaccuracies, launch condition variability and atmospheric factors and their relative influence on a guidance implementation package.
\end{abstract}

Next we present a 6 DOF modeling with various step-like thrust-curves while maintain the same total impulse delivered by the original motor. The influence of the step size on the range of the GRAD rocket is investigated together with the dispersion influence. We show that significant range increase can be obtained while using the same propulsion unit with a steplike thrust-curve modification. We also investigate the influence of the step-like thrust-curve on dispersion of such a rocket and the technological possibilities to implement our solution.

In the last part of the paper we present a terminal guidance concept $122 \mathrm{~mm}$ rockets. A preliminary requirement for IMU units to be used for terminal guidance is shown together with the general guidance algorithm for several trajectories. Performance expectations are shown through the analysis of the IMU units performance as well as flight dynamics of the $122 \mathrm{~mm}$ rockets.

Keywords: unguided, rocket, guidance, terminal, thrust, range, dispersion, interpolation, numerical, simulation, inertial

\section{Nomenclature}

$\mathrm{u}, \mathrm{v}, \mathrm{w} \quad$ velocity components in body frame [meters/second]

$\theta \quad$ pitch angle [radians]

$\varphi \quad$ roll angle [radians]

$\psi \quad$ yaw angle [radians]

$I_{1} \quad$ axial moment of inertia $\left[\mathrm{kg} \mathrm{m}^{2}\right]$

$I_{2}=I_{3} \quad$ lateral moments of inertia $\left[\mathrm{kg} \mathrm{m}^{2}\right]$

Research Scientist, Romanian Space Agency (ROSA), Address: Str. Mendeleev, Nr. 21 25, Bucharest, 010362, Romania, florin.mingireanu@ rosa.ro 
$q_{0}, q_{1}, q_{2}, q_{3}$ quaternions used to compose elements of the rotation matrix

$F_{x}, F_{y}, F_{z} \quad$ resultant force components on the $\mathrm{X}, \mathrm{Y}$ and $\mathrm{Z}$ axis [Newtons]

$p, q, r \quad$ angular velocities on the pitch, roll and yaw axis [radians/second]

$F_{a, p} \quad$ vector sum of propulsive and aerodynamic forces along the given axis

[Newtons]

$\omega_{x B}^{E} \quad$ angular velocity around $\mathrm{x}$ axis expressed in Earth reference frame

$\omega_{y B}^{E}$ angular velocity around y axis expressed in Earth reference frame

$\omega_{z B}^{E}$ angular velocity around $\mathrm{z}$ axis expressed in Earth reference frame

$M_{x}$ aerodynamic moment around $\mathrm{x}$ axis

$M_{y}$ aerodynamic moment around y axis

$M_{z}$ aerodynamic moment around $\mathrm{z}$ axis

$\rho$ local air density $\left[\mathrm{kg} / \mathrm{m}^{3}\right]$

$S \quad$ rocket cross surface $\left[\mathrm{m}^{2}\right]$

$\beta \quad$ sideslip angle [radians]

$\alpha \quad$ angle of attack [radians]

$C_{A} \quad$ axial force coefficient

$C_{y \beta}$ side force coefficient derivative with sideslip angle [1/radian]

$C_{N \alpha}$

$C_{l}$ normal force coefficient derivative with angle of attack [1/radian]

$C_{l p}$ rolling moment coefficient

rolling moment coefficient derivative with roll rate [1/radian]

$C_{m \alpha}$

C pitching moment coefficient derivative with angle of attack [1/radian]

$m \alpha$

$C_{n r}$ pitching moment coefficient derivative with angle of attack rate [1/radian]

$C_{m q}$ yawing moment coefficient derivative with yaw rate [1/radian]

$C_{n p}$ $v$ pitching moment coefficient derivative with pitch rate [1/radian] yawing moment coefficient derivative with pitch rate [1/radian] total velocity independent of reference frame [meters/second]

$L$ reference length [meters]

\section{Introduction}

Unguided rockets have long been used in the battlefields around the world. They are attractive because they are inexpensive and they can cover larger areas in short reaction times. Several countries developed unguided rockets with the launchers usually installed on purposely made or modified trucks. The rockets have ranges from $20 \mathrm{~km}$ to more than $60 \mathrm{~km}$ being able to be fired individually or in salvos. The typical unguided rocket uses a solid rocket motor with double base or composite propellant. However, their dispersion is quite large typically being around $5 \%$ of their range which for a typical $20 \mathrm{~km}$ range can be more than 1000 meters. This rather large dispersion renders these unguided rockets not useful for precision applications. Thus most of the tactics involving unguided rockets have been developed along the lines of using these rockets for discouragement of troops by covering the target areas with a hundreds of rockets. In the past decades an important objective was to re-convert the former unguided rockets to guided missiles by using terminal guidance systems as well as increasing their range mostly by changing their motors to more powerful ones. 
The advancement of MEMS technology opens us a potential direction to exploit in order to develop a high performance terminal guidance unit which coupled with an innovative method of increasing the range can lead to a completely new and attractive battlefield product.

\section{6 DOF Numerical Model}

We developed an in-house 6 DOF numerical simulation using realistic parameters for the $122 \mathrm{~mm}$ unguided rocket shown in Table 1.

Table $1.122 \mathrm{~mm}$ unguided rocket characteristics

\begin{tabular}{l|l}
\hline \hline \multicolumn{1}{c|}{ Item } & \multicolumn{1}{c}{ Value } \\
\hline \hline Caliber & $122 \mathrm{~mm}$ \\
\hline Initial mass & $67 \mathrm{~kg}$ \\
\hline Burnout mass & $46 \mathrm{~kg}$ \\
\hline Burning time & $1.7 \mathrm{~s}$ \\
\hline Length & $2.87 \mathrm{~m}$ \\
\hline Initial axial moment of inertia & $0.1499 \mathrm{kgm}^{2}$ \\
\hline Final axial moment of inertia & $0.1238 \mathrm{kgm}^{2}$ \\
\hline Initial lateral moment of inertia & $41.58 \mathrm{kgm}^{2}$ \\
\hline Final lateral moment of inertia & $33.83 \mathrm{kgm}^{2}$ \\
\hline Average thrust & $23600 \mathrm{~N}$ \\
\hline \hline
\end{tabular}

In order to maintain the generality of the 6 DOF model we develop all the equations in nonflat non-inertial Earth approximation. However for rather short ranges that we are dealing with (e.g.: $20 \mathrm{~km}$ ) we can use the flat Earth approximation without having errors that would affect the applicability of our results.

The translation equations in non-inertial, non-flat Earth approximation are given below and are identical with the ones presented in $[1,2]$ :

$$
\left[\begin{array}{c}
\dot{u} \\
\dot{v} \\
\dot{w}
\end{array}\right]=\frac{1}{m}\left[\begin{array}{c}
F_{x} \\
F_{y} \\
F_{z}
\end{array}\right]+g\left[\begin{array}{c}
-\sin \theta \\
\cos \theta \sin \varphi \\
\cos \theta \cos \varphi
\end{array}\right]+\left[\begin{array}{c}
\omega_{x B}^{E} \\
\omega_{y B}^{E} \\
\omega_{z B}^{E}
\end{array}\right]\left[\begin{array}{c}
u \\
v \\
w
\end{array}\right]
$$

The Earth's diurnal angular velocities are considered above basically as transport velocities for the transformation from one frame of reference to another.

For our purposes, we can neglect in our model the Earth's diurnal velocities due to small influences that they have onto typical trajectories of unguided rockets with ranges between $20 \mathrm{~km}$ and $70 \mathrm{~km}$. We can also assume yet another simplification of Earth being flat.

Hence, with the above simplifications of non-rotating, flat Earth approximation, the translational equations of motion in tensor notation form become [4]:

$m D^{B} v_{B}^{E}+m \Omega^{B E} v_{B}^{E}=F_{a, p}+m g$ 
We can write (2) in matrix coordinate form which makes them easier to be programmed on a computer:

$$
\left[\begin{array}{c}
\dot{u} \\
\dot{v} \\
\dot{w}
\end{array}\right]^{B}+\left[\begin{array}{ccc}
0 & -r & q \\
r & 0 & -p \\
-q & p & 0
\end{array}\right]^{B}\left[\begin{array}{l}
u \\
v \\
w
\end{array}\right]^{B}=\left[\begin{array}{c}
F_{x} / m \\
F_{y} / m \\
F_{z} / m
\end{array}\right]^{B}+\left[\begin{array}{lll}
t_{11} & t_{12} & t_{13} \\
t_{21} & t_{22} & t_{23} \\
t_{31} & t_{32} & t_{33}
\end{array}\right]^{B L}\left[\begin{array}{c}
0 \\
0 \\
m g
\end{array}\right]^{L}
$$

Next we show the rotational dynamics equations which provide the relation between the aerodynamic and propulsive moments and angular accelerations of the rocket. Through the double integration of the angular accelerations one can obtain the angular orientation in terms of pitch, yaw and roll angles, hence, obtaining the full attitude solution.

By applying Euler's law we have the following attitude dynamics equations in the body coordinates frame because the moments of inertia tensor has a simple and constant form in this reference system. In tensor form we have the following rotation equations:

$$
\left[\dot{\omega}^{B E}\right]^{B}=\left(\left[I_{B}^{B}\right]^{B}\right)^{-1}\left(-\left[\Omega^{B E}\right]^{B}\left[I_{B}^{B}\right]^{B}\left[\omega^{B E}\right]^{B}\left[m_{B}\right]^{B}\right)
$$

Given the body angular rates we can use equation (4) to obtain the body attitude relations through a set of 4 differential equations written in quaternion formulation:

$$
\left[\begin{array}{c}
\dot{q_{0}} \\
\dot{q_{1}} \\
\dot{q_{2}} \\
\dot{q_{3}}
\end{array}\right]=\frac{1}{2}\left[\begin{array}{cccc}
0 & -p & -q & -r \\
p & 0 & r & -q \\
q & -r & 0 & p \\
r & q & -p & 0
\end{array}\right]\left[\begin{array}{c}
q_{0} \\
q_{1} \\
q_{2} \\
q_{3}
\end{array}\right]
$$

The quaternions are related to the Euler angles through the following well-known relations in the aerospace field [3], [4]:

$$
\begin{aligned}
& q_{0}=\cos \left(\frac{\psi}{2}\right) \cos \left(\frac{\theta}{2}\right) \cos \left(\frac{\varphi}{2}\right)+\sin \left(\frac{\psi}{2}\right) \sin \left(\frac{\theta}{2}\right) \sin \left(\frac{\varphi}{2}\right) \\
& q_{1}=\cos \left(\frac{\psi}{2}\right) \cos \left(\frac{\theta}{2}\right) \sin \left(\frac{\varphi}{2}\right)-\sin \left(\frac{\psi}{2}\right) \sin \left(\frac{\theta}{2}\right) \cos \left(\frac{\varphi}{2}\right) \\
& q_{2}=\cos \left(\frac{\psi}{2}\right) \sin \left(\frac{\theta}{2}\right) \cos \left(\frac{\varphi}{2}\right)+\sin \left(\frac{\psi}{2}\right) \cos \left(\frac{\theta}{2}\right) \sin \left(\frac{\varphi}{2}\right) \\
& q_{3}=\sin \left(\frac{\psi}{2}\right) \cos \left(\frac{\theta}{2}\right) \cos \left(\frac{\varphi}{2}\right)-\cos \left(\frac{\psi}{2}\right) \sin \left(\frac{\theta}{2}\right) \sin \left(\frac{\varphi}{2}\right)
\end{aligned}
$$

Based on the above quaternions one can write the full transformation matrix from body to Earth reference frame: 


$$
[T]^{B L}=\left[\begin{array}{ccc}
q_{0}^{2}+q_{1}^{2}-q_{2}^{2}-q_{3}^{2} & 2\left(q_{1} q_{2}+q_{0} q_{3}\right) & 2\left(q_{1} q_{3}-q_{0} q_{2}\right) \\
2\left(q_{1} q_{2}-q_{0} q_{3}\right) & q_{0}^{2}-q_{1}^{2}+q_{2}^{2}-q_{3}^{2} & 2\left(q_{2} q_{3}+q_{0} q_{1}\right) \\
2\left(q_{1} q_{3}+q_{0} q_{2}\right) & 2\left(q_{2} q_{3}-q_{0} q_{1}\right) & q_{0}^{2}-q_{1}^{2}-q_{2}^{2}+q_{3}^{2}
\end{array}\right]
$$

Next we consider a reference frame connected to the missile and we also consider that the axis of the reference frame are aligned with the main axis of inertia of the missile.

Hence, we can express the Euler equations in simple scalar form for easier programming on the computer through the following relations:

$$
\begin{aligned}
& \dot{p}=\frac{1}{I_{1}}\left[\left(I_{2}-I_{3}\right) q r+M_{x}\right] \\
& \dot{q}=\frac{1}{I_{2}}\left[\left(I_{3}-I_{1}\right) p r+M_{y}\right] \\
& \dot{r}=\frac{1}{I_{3}}\left[\left(I_{1}-I_{2}\right) p q+M_{z}\right]
\end{aligned}
$$

The aerodynamic forces and moments [2] that appear in the above equations are given below with the full notations explained in the nomenclature section of this paper:

$$
\begin{aligned}
& F_{x}=\frac{1}{2} \rho v^{2} S\left(-C_{A}\right) \\
& F_{y}=\frac{1}{2} \rho v^{2} S\left(-C_{y \beta} \beta\right) \\
& F_{z}=\frac{1}{2} \rho v^{2} S\left(-C_{N \alpha} \alpha\right) \\
& M_{x}=\frac{1}{2} \rho v^{2} L\left(C_{l}+\frac{C_{l p} p L}{2 v}\right) \\
& M_{y}=\frac{1}{2} \rho v^{2} L\left[C_{m \alpha} \alpha+\left(C_{m \alpha}+C_{m q}\right) \frac{q L}{2 v}\right] \\
& M_{z}=\frac{1}{2} \rho v^{2} L\left[C_{n \beta} \beta+\left(C_{n r} r+C_{n p} p\right) \frac{L}{2 v}\right]
\end{aligned}
$$

The aerodynamic coefficients for the 122 rocket extracted from [1] and also verified with Missile DATCOM software package.

We also used the following simplifications due to $90^{\circ}$ rotational symmetry:

$$
\begin{aligned}
& C_{y \beta}=C_{N \alpha} \\
& C_{n \beta}=-C_{m \alpha} \\
& C_{n r}=C_{m q}
\end{aligned}
$$

Beside the characteristics of the rocket given in Table 2, the algorithm is initialized by giving the initial velocity vector which is typically $(0,0,0)$, the launch elevation angle and the angular velocities which are also typically $(0,0,0)$. 
Next the forces and aerodynamic moments are evaluated and inserted into the translational equations which are solved through Runge-Kutta 4. The output is the translational velocity in the body frame. By applying the quaternion rotation matrix we obtain the translational velocity vector in inertial reference frame and then we can find the new positions by integrating the velocities.

At the same time the velocity in the body frame serves as input in the forces and moments computation which through equations (8) give the attitude solution.

The entire algorithm is repeated until an end condition is met- typically until the rocket hits the ground which translates into $h=0$.

\section{Range Increase Through Step-Like Thrust Curve}

The range is one of the most important parameters of both an unguided and a guided weapon. More range usually offers more combat field capability to keep the launchers out of enemy's reach while maintaining battlefield superiority through precision strikes.

For unguided rockets the range increase is usually performed by replacing the current double base rocket motor with a composite rocket motor. However this converts directly into significant cost increase as well as technological modifications of launchers infrastructure due to higher combustion temperature of composite propellants which can lead to premature degradation of launch tubes.

We propose a method to increase the range of a given $122 \mathrm{~mm}$ unguided rocket by using its original rocket motor and modifying the thrust curve in order to decrease the overall drag force over the entire trajectory.

One of the limiting factors in the range of unguided rockets is the drag force that acts over the entire trajectory and decelerates the rocket with a factor proportional with the $\mathrm{v}^{2}$.

We modified a thrust curve of a motor by inserting a variable time delay either in the middle of the original thrust curve. By inserting the delay the average velocity of the rocket on the trajectory decreases, hence, decreasing the drag force and further increasing the attainable trajectory range.

We performed three numerical test cases with the following time delays:

- $\mathrm{dt}=0 \mathrm{~s}$ (standard motor) with a maximum drag force of $\sim 1510 \mathrm{~N}$

- $\mathrm{dt}=3.0 \mathrm{~s}$ with a maximum drag force of $\sim 1356 \mathrm{~N}$

- $\mathrm{dt}=6.0 \mathrm{~s}$ with a maximum drag force of $\sim 1152 \mathrm{~N}$

The drag force as a function of time for each of the delays is represented in Figures 1, 2 and 3 where the drag force is represented on the vertical axis and has the units in Newtons while the time is represented on the horizontal axis and has the units in seconds. 


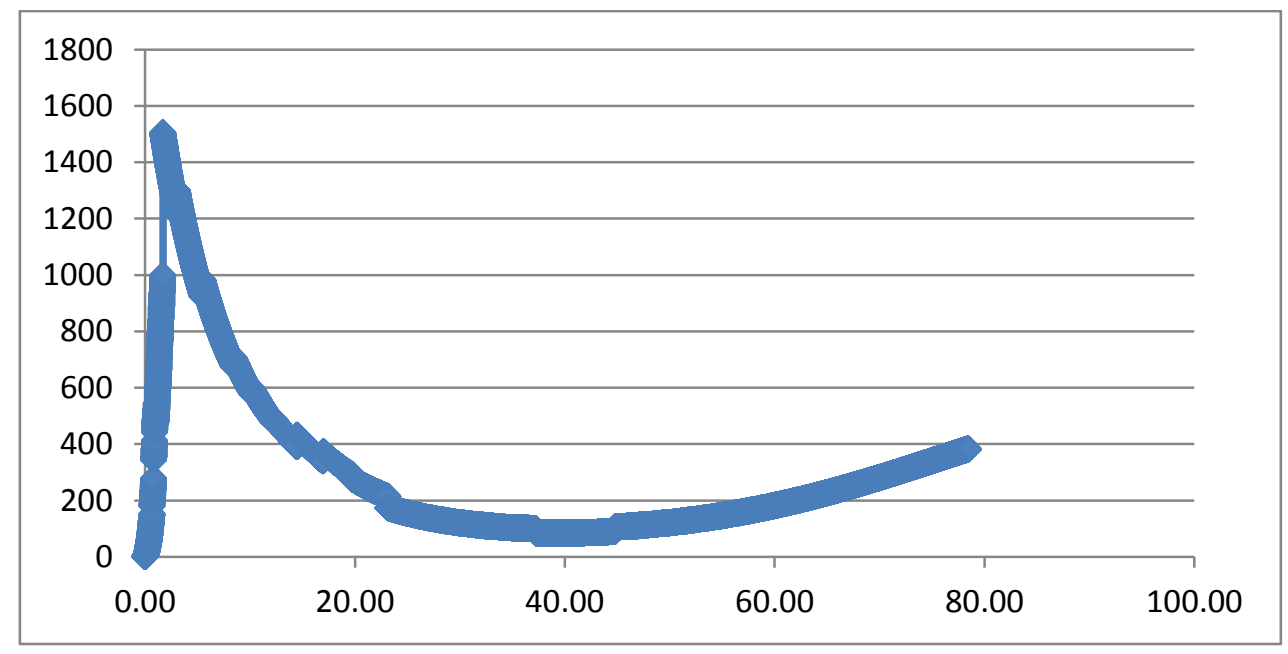

Fig.1 Drag force function of time $d t=0 \mathrm{~s}$

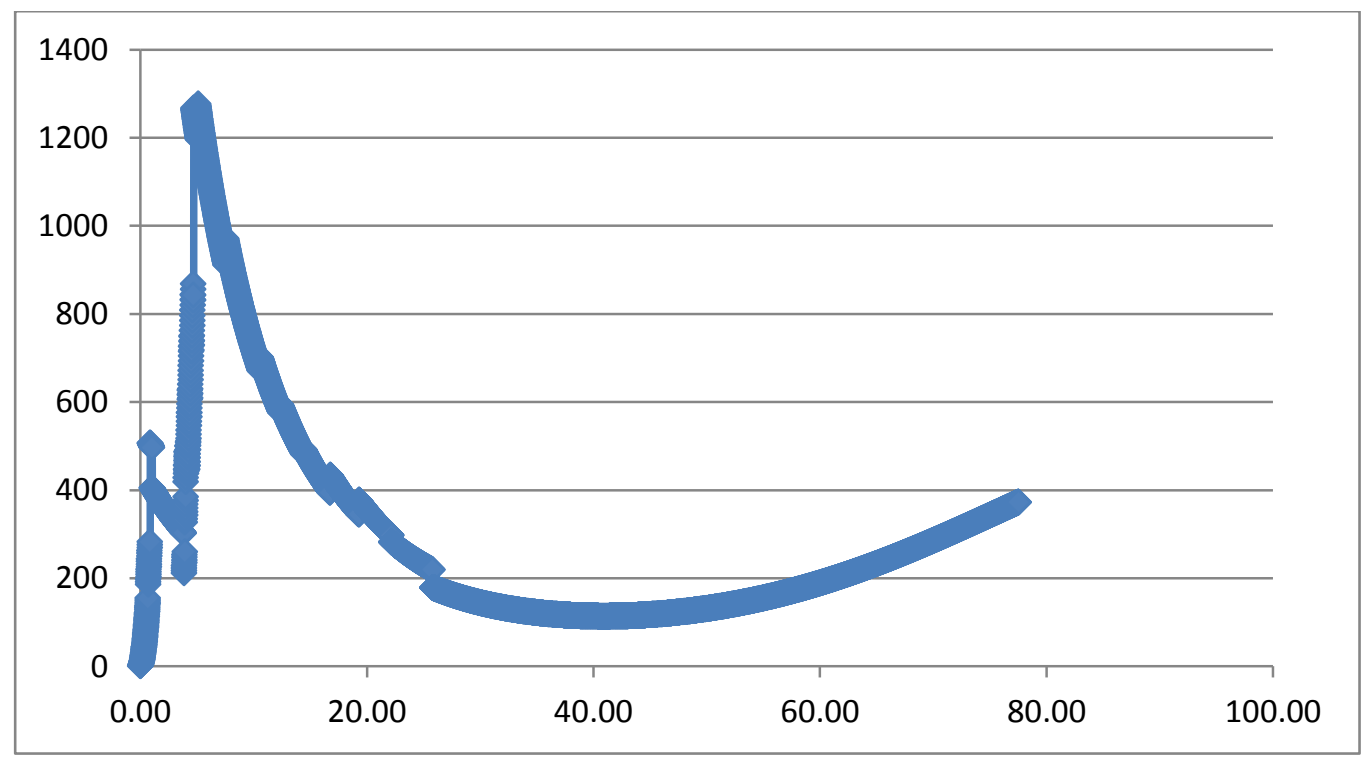

Fig.2 Drag force function of time $\mathrm{dt}=3 \mathrm{~s}$

At the same time the trajectory range is shown considering a typical $50^{\circ}$ inclination of the launcher in Figures 4 and 5 for the cases $d t=0 \mathrm{~s}$ and $d t=3 \mathrm{~s}$. In Figures 4 and 5 we have altitude in meters on the vertical axis and range also in meters on the horizontal axis.

For $\mathrm{dt}=0 \mathrm{~s}$ the range obtained is $\sim 20168$ meters while for $\mathrm{dt}=3 \mathrm{~s}$ the range is $\sim 24443$ meters. 


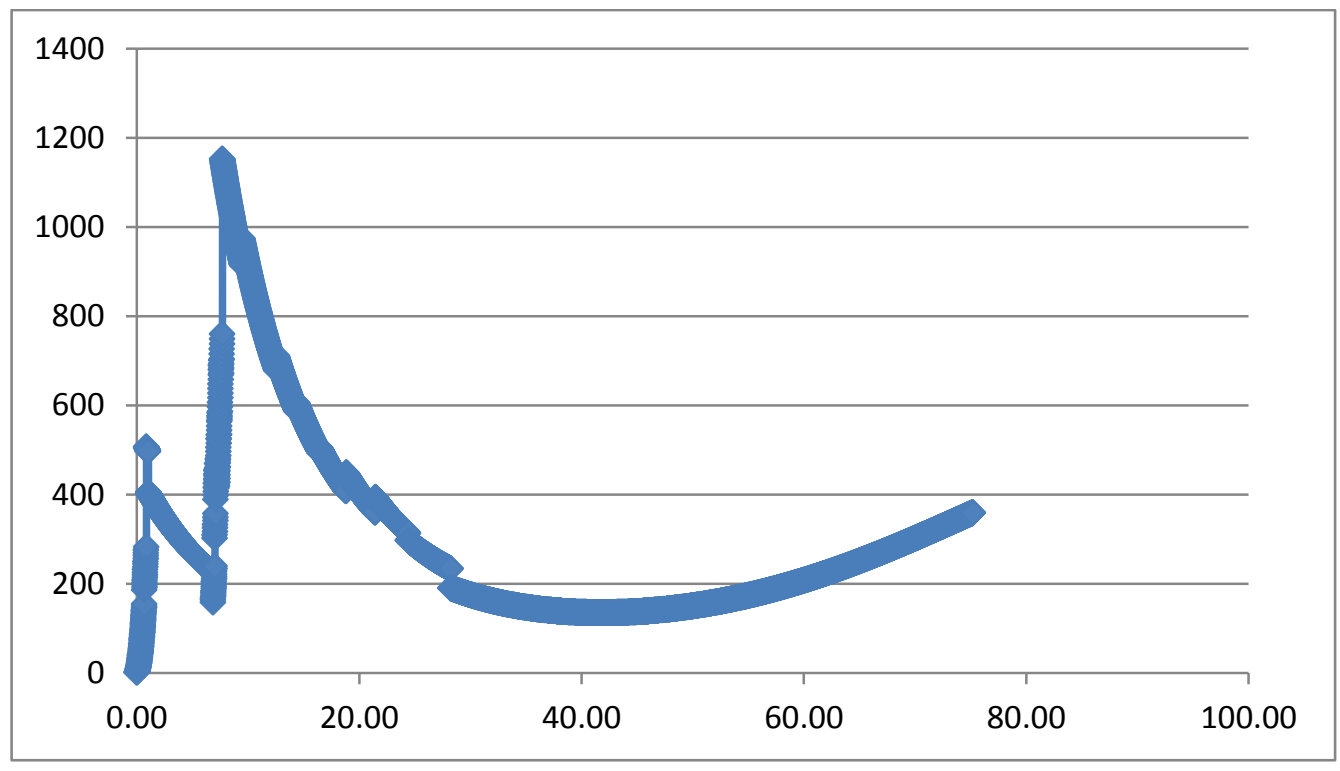

Fig.3 Drag force function of time $\mathrm{dt}=6 \mathrm{~s}$

Hence, we can observe, with all the aerodynamic simplifications and idealistic assumptions that the range can indeed be increased through the modification of the thrust curve of a given propulsion unit. For our numerical cases the range increase was $\sim 4275$ meters which is $\sim 21 \%$ of the standard range obtained without any thrust curve modification.

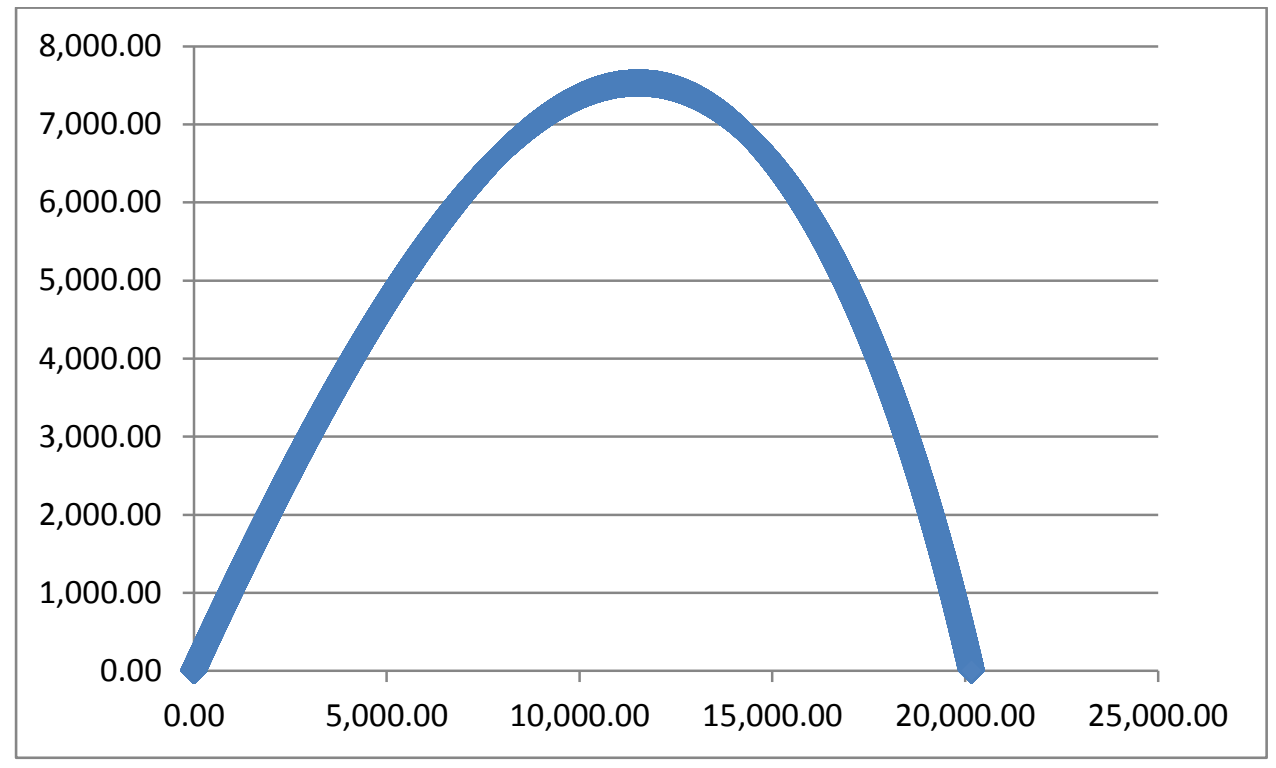

Fig.4 Trajectory for $\mathrm{dt}=0 \mathrm{~s}$ 


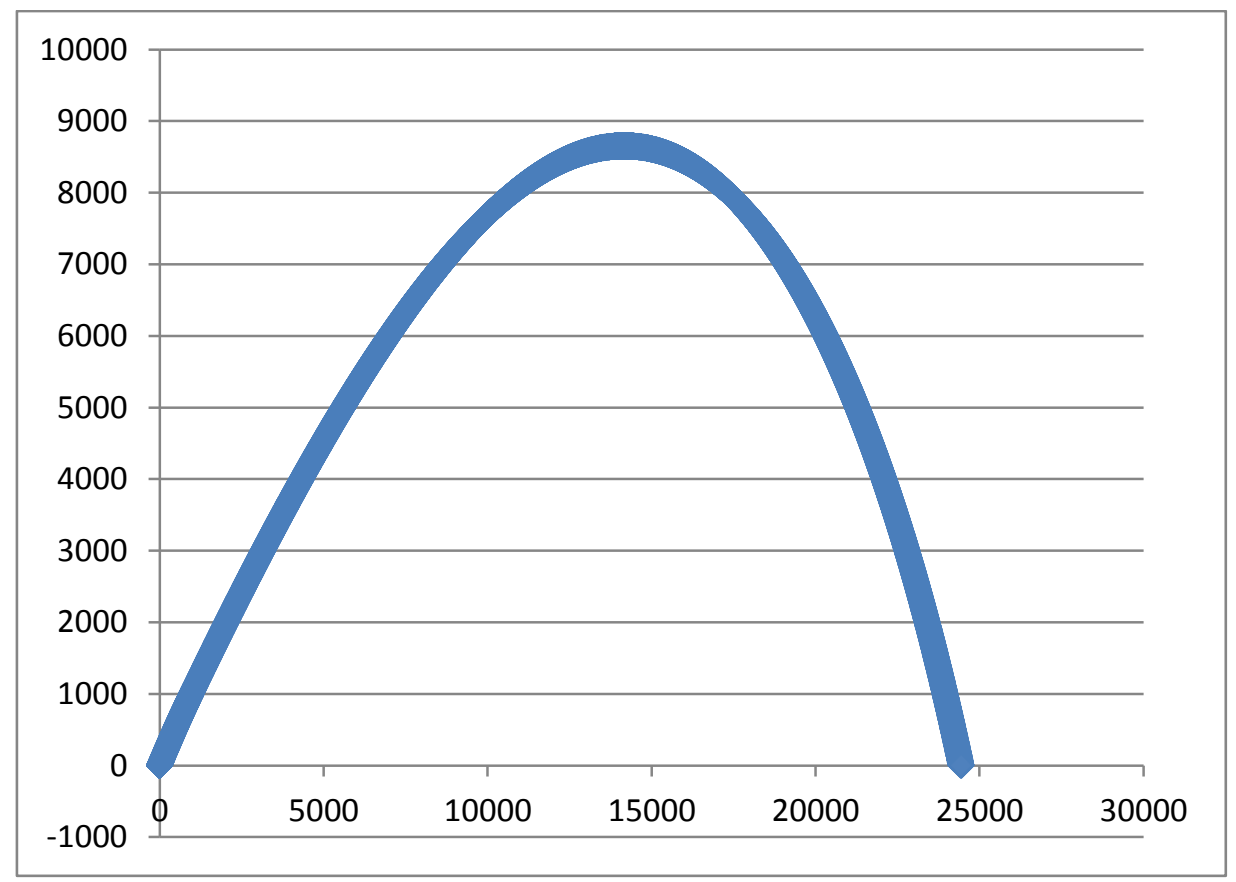

Fig.5 Trajectory for $\mathrm{dt}=3 \mathrm{~s}$

The general trend for the range is that it increases with the increase of the time delay. An important note to make is that the time delay cannot be increased to very large results because this would lead to very low airspeeds at the end of the time delay which in turn decreases the overall dynamical stability of the considered rocket. A typical minimum acceptable airspeed at the end of the time delay is $200 \mathrm{~m} / \mathrm{s}$ which gives sufficient stability margin for the rocket to perform safely and predictable for the rest of the flight trajectory. Technologically we propose to implement the time delay through the usage of an electronic programmable unit which can be programmed wireless for a variety of values of the time delay. Hence, by programming different the time delays one can change the ranges that the rocket can reach. All this programming can be done on the field, real time through the usage of a PDA ruggedized device which is in standard use within the military forces.

\section{Low Cost IMU Terminal Guidance Unit}

Besides the range, the dispersion of the trajectories is yet another important parameter that characterizes the performances of an unguided rocket system. The dispersion of trajectories can have multiple causes which can be divided into two large families:

- natural dispersion: caused by mainly meteorological effects (e.g.: wind, air density fluctuations, temperature variations etc.)

- artificial dispersion: caused mainly by manufacturing imperfections (e.g.: fins misalignment, thrust misalignment, variations of rocket motor performance, variations of rocket mass etc.). 
Both of these families of dispersion causes generate trajectory deviation from the standard predicted trajectory. By diminishing the artificial dispersion causes as much as possible, through implementing a more stringent quality control, one can increase the quality of rocket projectiles. However, one cannot eliminate any of the above causes and hence additional means of guidance have to be implemented in order to correct the deviations introduced in the trajectory by the above causes.

The advancements of MEMS technology especially in the field of sensors (e.g.: rate gyroscopes, accelerometers) produced the technological capability to implement small IMUs in packages that can realistically integrated within typical $122 \mathrm{~mm}$ rockets.

In Figure 4 we presented a typical trajectory for a $122 \mathrm{~mm}$ rocket launched at $50^{0}$ inclination with a standard solid rocket motor with no thrust-curve modification. The range is 20168 meters.

In Figure 7 we present a typical trajectory in which the rocket has to reach a target which is placed at $18 \mathrm{~km}$, considering that the launch inclination is left unchanged at $50^{\circ}$. The steering is performed after the rocket reaches the apex of the trajectory and mainly consists in reducing the range assuming correct azimuth. This can be observed in the Figure 7 by a steeper descent portion of the trajectory than on the Figure 4 where no steering is used.

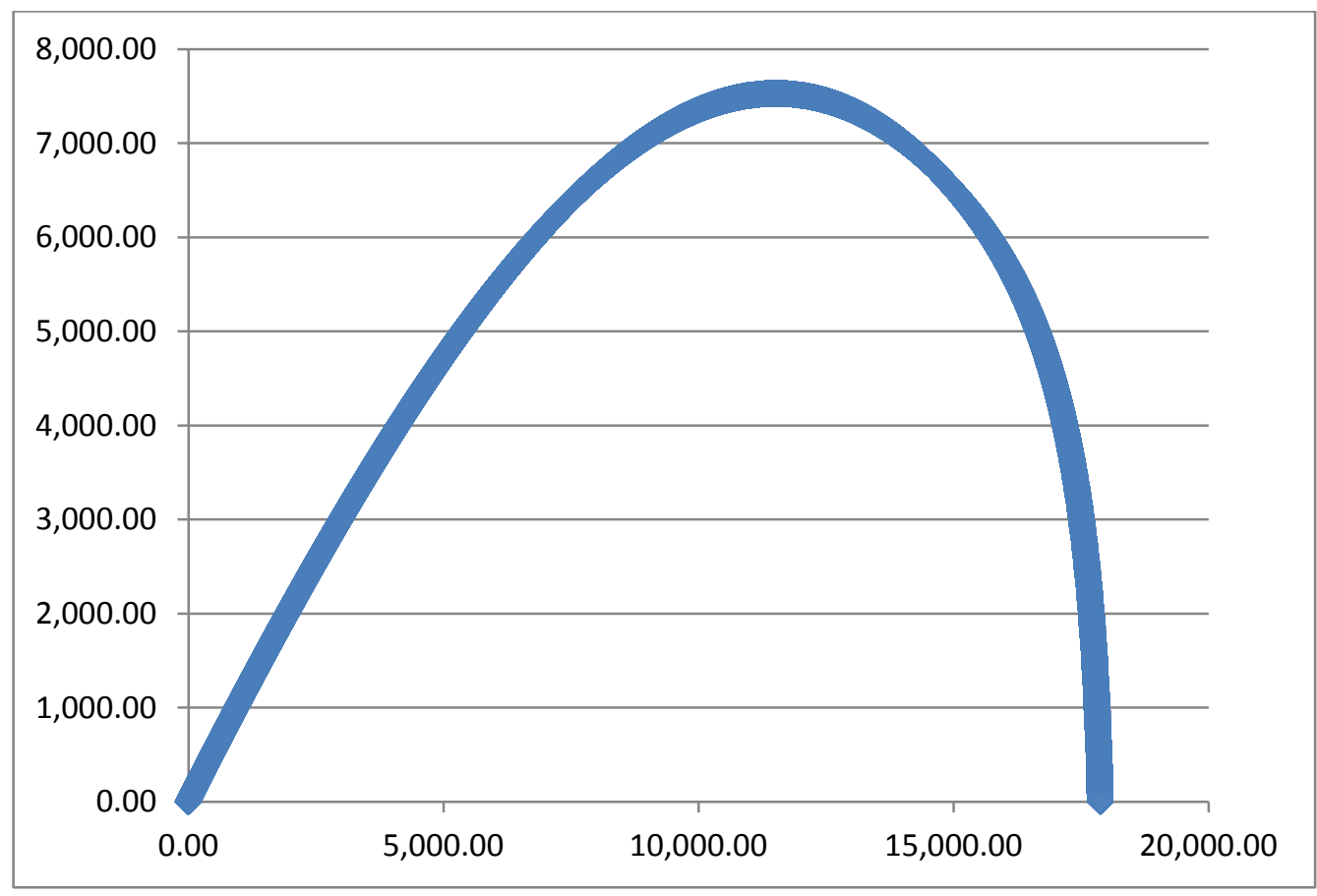

Fig. 6 Steering towards an $18 \mathrm{~km}$ target

The general conceptual schematic of the autopilot is given in Figure 7. 


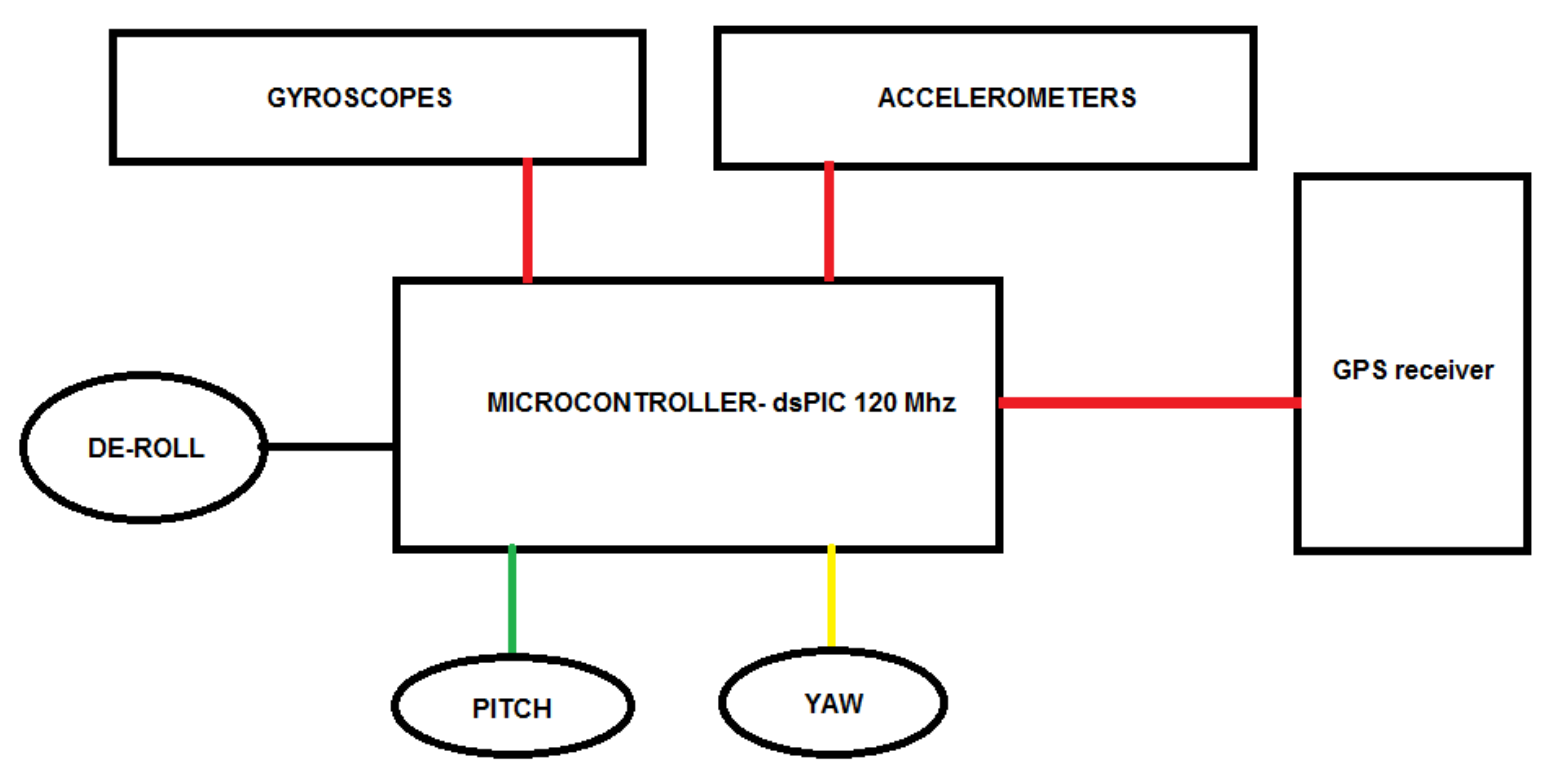

Fig. 7 Autopilot conceptual schematics

We basically use 4 rate gyroscopes in order to increase their apparent sampling rate. At the same time we use 4 accelerometers which are averaged and filtered through a simple add and drop filter. The microcontroller gets the information from the sensors and then by Kalman filtering the information with the GPS information reports the attitude and position solution of the rocket.

An important detail is that the autopilot is not activated until the burnout because the high launching acceleration would impose too large requirements for the low cost MEMS components that are to be used.

The guidance algorithm is based on a series of events that ensure the proper operation of all sensors and the proper steering commands in order to have as small as possible impact error.

Just before take-off the GPS is initialized and the lock position validity is checked. Upon successfully passing the GPS validity check the system removes failsafe from the motor igniter. Once the motor is ignited, the autopilot just maintains itself in standby without doing anything. An axial mounted high-g accelerometer determines the motor burnout and 2 seconds later issues enable command to autopilot to begin de-roll procedure while performing a pre-callibration on the guidance gyroscopes and accelerometers. Throughout the de-roll procedure the readings from a high-rate gyroscope are used and the autopilot and warhead section are de-rolled the rocket to $0 \mathrm{~Hz}$. The rest of the rocket keeps rolling through the usage of a de-roll bearing.

Next, the autopilot initializes the GPS and after receiving valid position it enables the full attitude and positioning portion of the code based on high fidelity MEMS rate-gyroscopes and accelerometers. Ideally this is performed before the rocket reaches $2 / 3$ of the maximum altitude. 
After passing the apogee of the trajectory the autopilot transmits the commands to a series of fins placed in the nose of the rocket. Basically the fins generate aerodynamic moments around pitch, yaw, roll axis, hence, steering the entire rocket.

As it can be seen, the general guidance algorithm is initiated after the trajectory apex extending by numerical simulation the predicted impact point (PIP). The errors between the current predicted impact point and the desired impact point both on longitudinal and lateral axis are fed into the PID loops into the autopilot adequately steering the rocket. Next, the entire procedure is repeated until the rocket impacts the ground. Usually the rocket needs $1 / 3$ of the entire descent time to correct and deviations generated until the trajectory apex.

\section{Conclusions}

The unguided rockets can still be attractive for a number of applications given that some upgrades are performed both in the area of increasing the range as well as in the area of decreasing the dispersion.

We showed an original method of increasing the range by implementing a step-like thrust curve which decreases the average airspeed which in turn decreases the drag force leading to an increase of the range.

We also showed the concept of a low cost IMU-based autopilot which serves for the terminal guidance of an originally unguided $122 \mathrm{~mm}$ rocket projectile. PID guidance loop on both pitch and yaw is shown to be adequate for the terminal guidance application as well as initial de-rolling procedure in order to decrease the angular stress on the low cost MEMS rate gyroscopes.

Future work will include Monte Carlo analysis of dispersion parameters as well as non-central and multi-pause thrust-curves for even more potential of range increase.

\section{References}

[1] M. Khalil, H. Abdalla* and O. Kamal, "Trajectory Prediction for a Typical Fin Stabilized Artillery Rocket" ", Proceedings of the $13^{\text {th }}$ International Conference on Aerospace Sciences and Aviation Technology, ASAT-13, ” May 26-28, 2009

[2] Pawat Chusilp, Weerawut Charubhun, and Navapan Nutkumhang. „A Comparative Study on 6-DOF Trajectory Simulation of a Short Range Rocket using Aerodynamic Coefficients from Experiments and Missile DATCOM", The Second TSME International Conference on Mechanical Engineering, 19-21 October, 2011, Krabi

[3] Design of Aerodynamically Stabilized Free Rocket (1990). MIL-HDBK-762, US Army Missile Command, AL, USA

[4] Peter Zipfel, "Modeling and Simulation of Aerospace Vehicle Dynamics", AIAA, 2000, ISBN 1-56347-456-5 